\title{
CLOSED-LOOP CONTROL TECHNIQUES FOR ACTIVE VIBRATION SUPPRESSION OF A FLEXIBLE MECHANICAL SYSTEM
}

\author{
Ing. Vladímir Villaverde Huertas, prof. Ing. Boris Rohal'-Ilkiv, Csc.
}

Institute of Automation, Measurement and Applied Informatics, Faculty of Mechanical Engineering, Slovak University of Technology in Bratislava, Námestie Slobody 17, 81231

Bratislava, Slovak Republic

Vladimir.huertas@stuba.sk, boris.rohal-ilkiv@stuba.sk

Keywords: subspace identification, explicit predictive controller, positive position feedback, piezoelectric actuation

\begin{abstract}
This paper investigates the problem of vibration attenuation of a lightly damped mechanical system using piezoelectric actuation. First of all, an explicit predictive controller will be designed using the Matlab multi-parametric toolbox. Then, we will explore the positive position feedback technique and test the discrete-time PPF controller using an xPC target real-time system. On the other hand, we will realize the modal analysis of the analyzed flexible system in order to determine the frequency corresponding to the first mode shape. This frequency will be utilized as PPF controller frequency. Moreover, the state-space model of the flexible mechanical system will be obtained using the Matlab system identification toolbox applying the subspace identification approach.
\end{abstract}

\section{INTRODUCTION}

Flexible structures are commonly used in a wide variety of engineering applications, which are characterized by several resonant modes. For instance, some typical flexible structures are widely used in applications such as aircraft, bridges, buildings, etc. Therefore, it is crucial to damp the flexible structure vibrations in an effective manner since they may affect the stability and performance of the structures [3], [4]. For this reason, vibration damping technologies are needed to dissipate mechanical energy related to motion in mechanical systems.

There are many active control strategies that have been successfully implemented in practice to actively control the vibration of flexible structures. In this paper, we are going to develop two controllers for vibration suppression of a cantilever beam (Fig. 1). An explicit MPC controller will be designed first. For this purpose, it is necessary to obtain the state-space representation of the system. According to this, we are going to use the subspace identification method in frequency domain applying the fast Fourier transformation to obtain the state space model for the flexible structure. Moreover, we will use a Kalman filter in order to estimate the system state.

Lastly, we will design a positive position feedback controller. The PPF control strategy developed by Goh and Caughey uses a second-order filter to compensate the position signal. A good reason for using the positive position feedback (PPF) is the feasibility of controlling several 
modes simultaneously, keeping global stability conditions. On the other hand, the PPF controller accomplishes its best performance if tuned properly to the characteristics of the structure to be controlled. Therefore, it is necessary to determine its three important parameters (i.e. controller frequency, damping ratio and gain).

\section{SUBSPACE IDENTIFICATION OF THE FLEXIBLE MECHANICAL SYSTEM}

This section deals with estimating a state-space model of a cantilever beam (see Fig. 1) using the subspace identification method based on output and input data records.

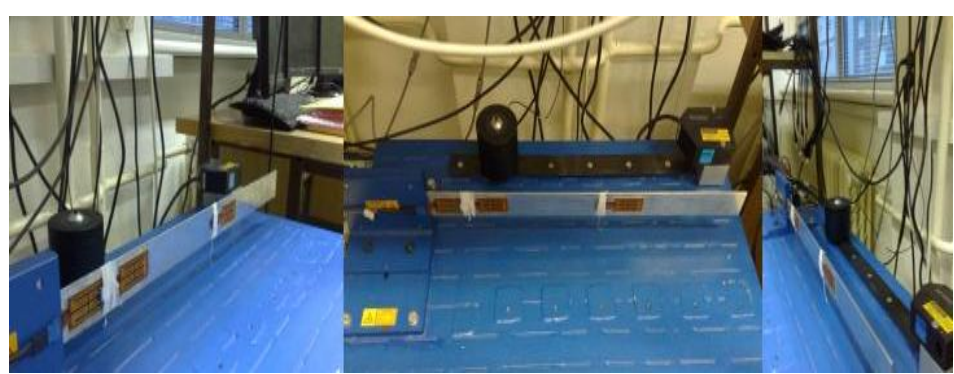

Figure 1: Cantilever beam.

For this purpose, we have used a chirp signal as a piezoelectric actuator input. This signal increases its frequency from 0.1 to 100 [Hz]. On the other hand, the tip displacement of the flexible beam has been considered as the system output.

It is necessary to highlight that the subspace identification approach estimates the states directly from input-output data first, then the system matrices can be obtained [2]. While the classical identification method obtains the system matrices first, and then the states can be estimated.

It is important to take into account that when modeling flexible structures, it is common to fit the input and output data in the frequency domain. For this reason, we have used the Fast Fourier Transform (FFT). Fig. 2 shows the data in the frequency domain. 


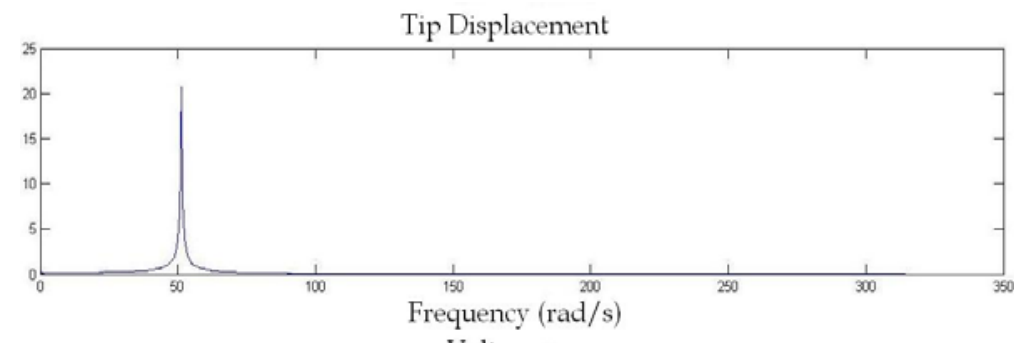

Voltage

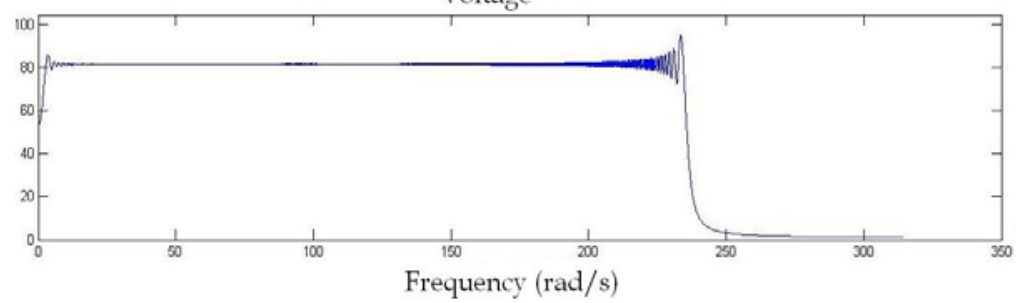

Figure 2: Measured data for identification purposes.

In this paper, we have used the Matlab system identification toolbox, which is an analysis module that contains necessary tools for building mathematical models of dynamical systems. Therefore, using the subspace identification approach, we could obtain the following state-space model for the analyzed flexible mechanical system (see Fig. 1).

$$
\begin{aligned}
& x(k+1)=A x(k)+B u(k) \\
& y(k)=C x(k)+D u(k) \\
& A=\left(\begin{array}{ll}
0.8692 & -1.119 \\
0.2136 & 0.8694
\end{array}\right) \quad B=\left(\begin{array}{c}
0.001626 \\
-0.0004816
\end{array}\right) \\
& C=\left(\begin{array}{ll}
-0.5513 & -0.7061)
\end{array} \quad \mathrm{D}=0\right.
\end{aligned}
$$

Eq. (1) is called the state equation, and the vector $x$, the state vector, contains the state variables. Eq. (2) is known as the output equation and the $A, B, C, D$ matrices are called the system, input, output and feed-forward matrices, respectively.

Fig. 3 shows a comparison between the estimation data and the obtained model. It is important to remark that a sample time $\mathrm{Ts}=0.01$ has been considered. 


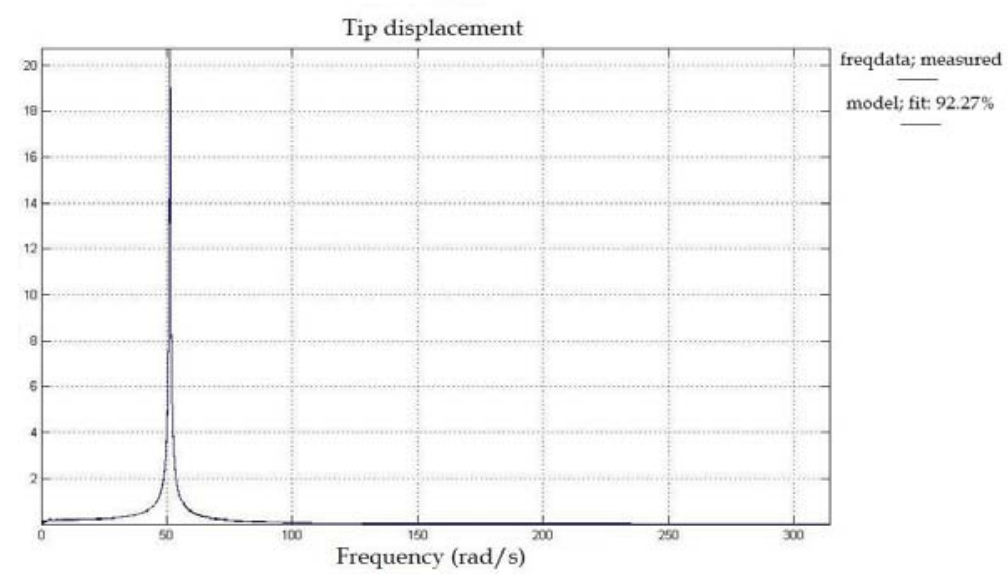

Figure 3: Estimation data vs obtained model.

\section{EXPLICIT PREDICTIVE CONTROLLER DESIGN FOR VIBRATION ATTENUATION OF THE FLEXIBLE SYSTEM}

In this section we are going to use the Matlab multi-parametric toolbox in order to obtain an explicit MPC controller for vibration attenuation of the analyzed cantilever beam. The goal of this toolbox is to calculate feedback controllers for those constrained optimal control problems. In this sense, a quadratic optimization problem is solved off-line via multi-parametric programming. Thus, the solution has the form of a state feedback control law. Moreover, the state space is divided into polyhedral sets and for each of those sets the optimal control law is given as one affine function of the state [5]. Therefore, it is important to remark that the online speed depends on a number of control law regions. Hence, the online evaluation is reduced to point location and correspondence of the affine function.

To obtain the explicit MPC controller for vibration suppression of the lightly damped cantilever beam, we are going to utilize the state-space model achieved using the subspace identification approach. It was also necessary to provide suitable penalties on states and inputs.

Fig. 4 shows the achieved controller regions and the corresponding control law. 

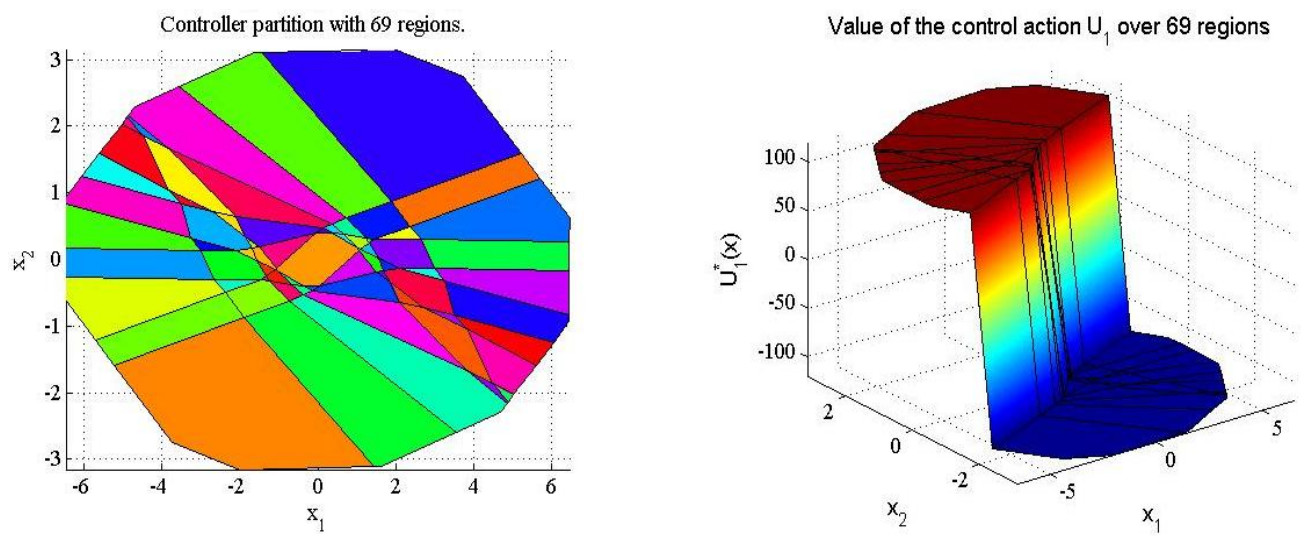

Figure 4: Controller regions (left) and control law (right).

Now, we will design a discrete-time control system to test the explicit MPC controller. In order to estimate the state of the system, it is necessary to analyze the observability, which is a necessary and sufficient condition for the existence of the observer [1]. The state estimation was done using a Kalman filter.

Fig. 5 shows the simulation results of the discrete-time control system for vibration attenuation of the cantilever beam.

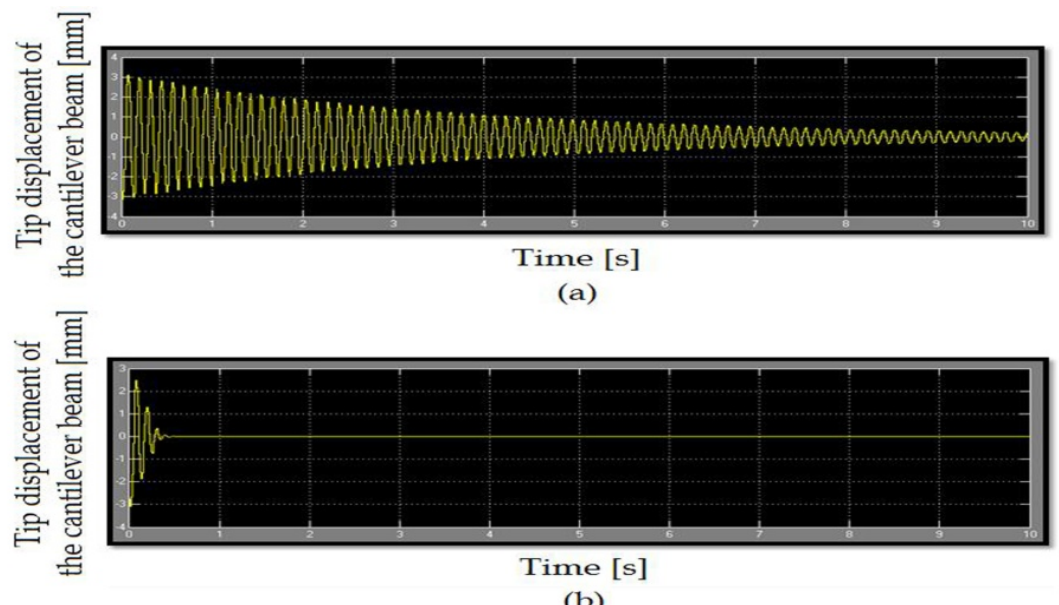

(b)

Figure 5: (a) Free response. (b) Closed-loop response.

\section{DISCRETE-TIME POSITIVE POSITION FEEDBACK CONTROLLER DESIGN}

In order to design a PPF controller we are going to consider an idealized modal model of the given flexible structure. Thus, a single-degree-of-freedom with viscous damping $\mathrm{c}$, stiffness $\mathrm{k}$ and mass $\mathrm{M}$ may be driven by an external force U. Using Lagrange method we may obtain the equation of motion for the structure, which characterizes its behaviour.

Lagrange's equation 


$$
\begin{aligned}
& \frac{d}{d t}\left(\frac{\partial E_{k}}{\partial \dot{q}_{j}}\right)-\frac{\partial E_{k}}{\partial q_{j}}+\frac{\partial E_{p}}{\partial q_{j}}+\frac{\partial D}{\partial \dot{q}_{j}}=Q_{j} \\
& j=1,2, \ldots
\end{aligned}
$$

where $E_{K}$ is the system kinetic energy function, $E_{p}$ stands for the system potential energy function, $D$ is the system dissipation function (damping function) and $Q_{j}$ stands for the generalized force along the generalized coordinate $q_{j}$.

Structure motion equation

$$
\ddot{x}+2 \zeta \omega \dot{x}+\omega^{2} x=f+u
$$

For implementing the PPF control technique, it is necessary to define $u$ as follows

$$
u=g \omega_{c}^{2} x_{c}
$$

Once we found the equation of motion for the analyzed flexible structure, we are going to formulate the PPF controller taking advantage of the modal equation form (4)

PPF controller

$$
\ddot{x}_{c}+2 \zeta_{c} \omega_{c} \dot{x}_{c}+\omega_{c}^{2} x_{c}=g \omega_{c}^{2} x
$$

where $g$ is the controller gain, $\omega_{\mathrm{c}}$ represents the controller frequency and $\zeta_{\mathrm{c}}$ stands for the controller damping ratio. On the other hand, taking the Laplace transform of each term in Eq. (6) we can express the transfer function of the PPF controller as follows

$$
G_{p p f}(S)=\frac{g \omega_{c}^{2}}{S^{2}+2 \zeta_{c} \omega_{c} S+\omega_{c}^{2}}
$$

To formulate a discrete-time PPF controller we are going to use the numerical backward difference approximation technique. Applying this technique we can find a suitable mathematical relationship between the s-domain and z-domain. It is very important to remark that the dynamic behaviour of the discrete-time control system depends on the sampling period. In other words, a change in the sampling period $T$ modifies the pole and zero locations in the $z$ plane [1]. The discrete-time PPF controller has the following form

$$
\operatorname{Gdppf}(\mathrm{z})=\frac{g \omega_{c}^{2} T^{2}}{\left(1+2 \zeta_{c} \omega_{c} T+\omega_{c}^{2} T^{2}\right)-2\left(1+\zeta_{c} \omega_{c} T\right) z^{-1}+z^{-2}}
$$

The controller frequency has been found from the modal analysis of the cantilever beam using Ansys.

Fig. 6 shows the frequencies corresponding to each vibration mode of the lightly damped cantilever beam and the first mode shape, which will be damped using the PPF controller. Note 
that the first mode frequency is 8.1578 [Hz]. This parameter will be used as the discrete-time PPF controller frequency.

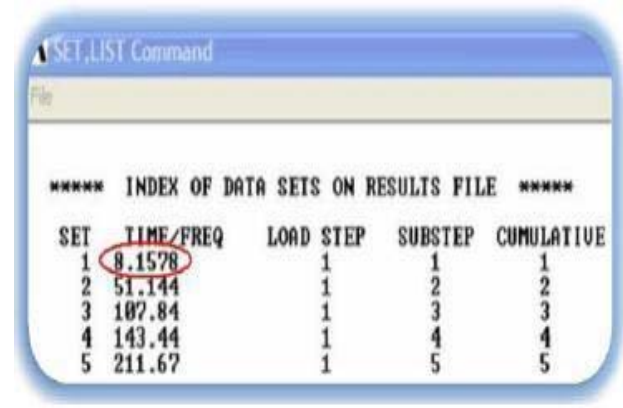

(a)

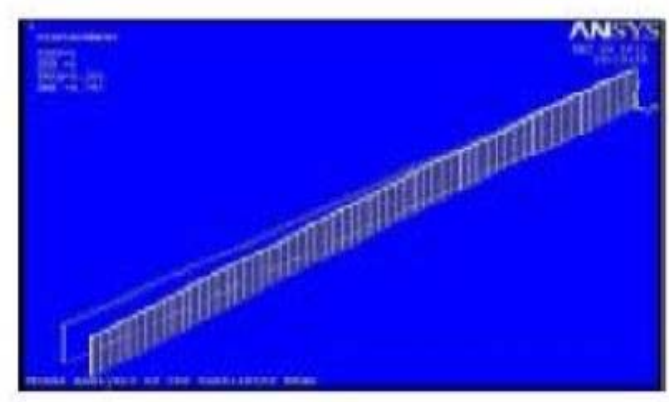

(b)

Figure 6: (a) Mode frequencies. (b) First mode shape.

Lastly, we have tested in real time the designed discrete-time PPF controller performance on the cantilever beam shown in Fig. 1. The piezoelectric patch closer to the clamped beam edge (see Fig. 1) was used as an actuator, which has been connected to an amplifier. Moreover, the tip displacement of the beam (slight deflection from its equilibrium position) has been measured utilizing a laser displacement sensor (KEYENCE LK-G82 laser triangulation device, see Fig. 1). It is very important to remark that the beam has been controlled using an xPC Target real-time system where the target PC interacts with the host PC utilizing the TCP/IP protocol. A National Instruments PCI DAQ data acquisition card installed on the target PC has been used in this work. The experiment ??? closed-loop block diagram is shown in Fig. 7.

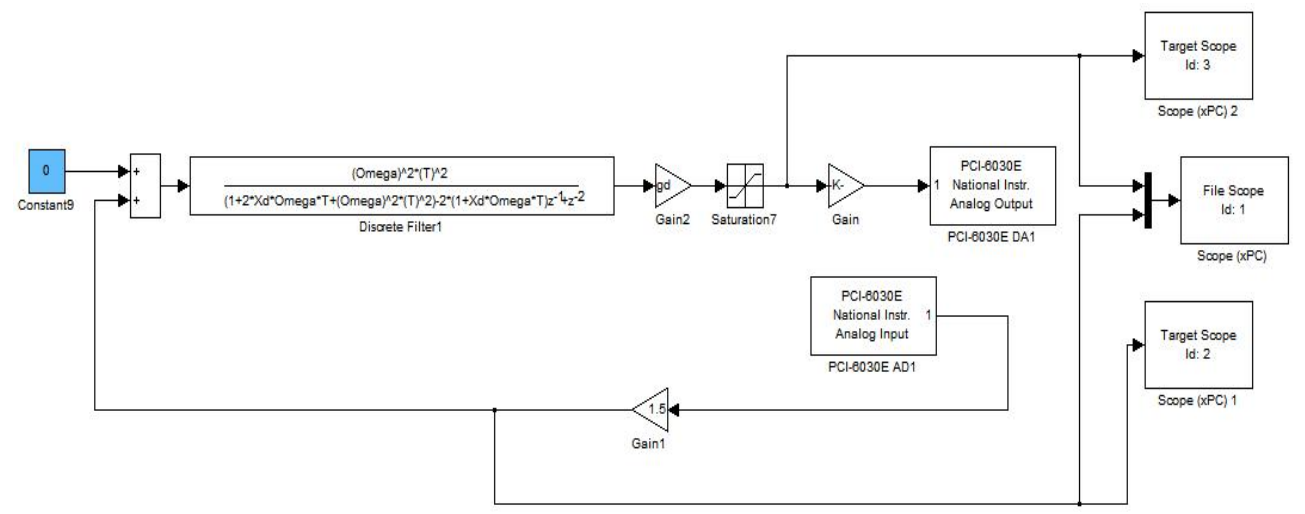

Figure. 7: Closed-Loop control system for vibration attenuation of the cantilever beam.

Finally, Fig. 8 shows the obtained experimental results. 


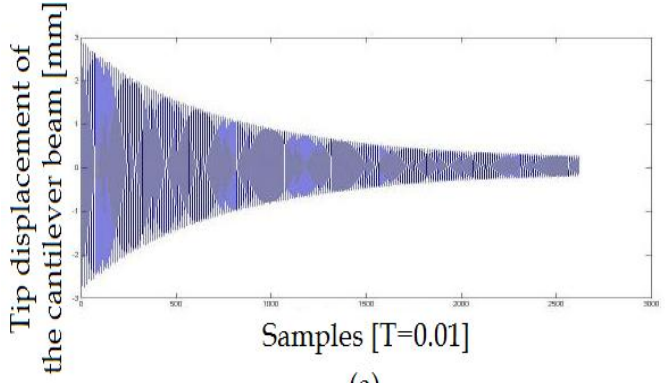

(a)

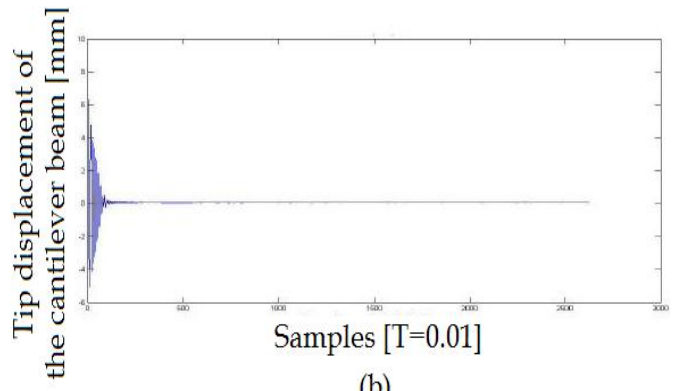

(b)

Figure 8: (a) Free response. (b) Closed-loop response.

\section{CONCLUSION}

This work has investigated the feasibility of using piezoelectric actuation for vibration suppression of a flexible system. For this purpose, a cantilever beam has been analyzed. The method of positive position feedback has demonstrated to be a quite useful technique for vibration attenuation in flexible structures. The discrete-time PPF controller was tuned choosing convenient values for controller parameters (i.e., damping ratio and gain). Moreover, the cantilever beam test structure was modeled using Ansys in order to find the structural frequency corresponding to the first mode, which was used as a filter frequency for completing the controller tuning.

On the other hand, we have demonstrated that the explicit MPC controller designed using the Matlab multi-parametric toolbox is very useful for vibration suppression of flexible mechanical systems. The design of this controller was done utilizing the obtained state-space model using the subspace identification approach. It was also necessary to provide the input and output constraints and the corresponding penalties.

\section{ACKNOWLEDGMENTS}

The research reported in this paper was supported by Slovak Grant Agency APVV, projects ID: APVV-0090-10 and APVV-0280-06. The supports are very gratefully appreciated.

\section{REFERENCES}

[1] OGATA, K.: Discrete-time control systems. Prentice-Hall. New Jersey. 1995.

[2] LJUNG, L.: System identification: Theory for the user. Prentice Hall, Upper Saddle River, NJ, USA. 1999. Second Edition.

[3] VILLAVERDE, V., ROHAL-ILKIV, B.: Discrete-time H-infinity controller design for vibration suppression of a flexible system. $10^{\text {th }}$ International conference PROCESS CONTROL 2012, Kouty nad Desnou, Czech Republic.

[4] PREUMONT, A.: Mechatronics - Dynamics of electromechanical and piezoelectric systems. Springer. Dordrecht, The Netherlands. 2006. 